(c) ACM, 2019. This is the author's version of the work. It is posted here by permission of ACM for your personal use. Not for redistribution. The definitive Trations on Multimedia Computing, Communications and Applications 15(4), article 108 https://doi.org/10.1145/3357333

\title{
Embedding distortion analysis in wavelet-domain watermarking
}

\author{
DEEPAYAN BHOWMIK, University of Stirling, United Kingdom \\ CHARITH ABHAYARATNE, University of Sheffield, United Kingdom
}

Imperceptibility and robustness are two complementary fundamental requirements of any watermarking algorithm. Low strength watermarking yields high imperceptibility, but exhibits poor robustness. High strength watermarking schemes achieve good robustness but often infuse distortions resulting in poor visual quality in host image. This paper analyses the embedding distortion for wavelet based watermarking schemes. We derive the relationship between distortion, measured in mean square error (MSE), and the watermark embedding modification and propose the linear proportionality between MSE and the sum of energy of the selected wavelet coefficients for watermark embedding modification. The initial proposition assumes the orthonormality of discrete wavelet transform. It is further extended for non-orthonormal wavelet kernels using a weighting parameter, that follows the energy conservation theorems in wavelet frames. The proposed analysis is verified by experimental results for both non-blind and blind watermarking schemes. Such a model is useful to find the optimum input parameters, including, the wavelet kernel, coefficient selection and subband choices for wavelet domain image watermarking.

CCS Concepts: • Security and privacy $\rightarrow$ Digital rights management.

ACM Reference Format:

Deepayan Bhowmik and Charith Abhayaratne. 2019. Embedding distortion analysis in wavelet-domain watermarking. $A C M$ Trans. Multimedia Comput. Commun. Appl. 0, 0, Article 0 ( 2019), 23 pages. https://doi.org/00.000/000.0000

\section{INTRODUCTION}

As digital technologies have shown a rapid growth within the last decade, content protection now plays a major role within content management systems where digital watermarking provides a robust and maintainable solution to enhance media security. The visual quality of host media, i.e., imperceptibility and robustness are widely considered as the two main properties vital for digital watermarking systems. They are complimentary to each other and hence challenging to attain the right balance between them. This paper proposes a model for estimating embedding distortion due to use of various wavelet kernels in watermarking algorithms. The model will be useful in designing new wavelet based watermarking algorithms with improved imperceptibility and robustness.

Frequency domain watermarking, more precisely wavelet-based watermarking, methodologies are highly favoured in the current research era. The wavelet domain is also compliant within many image coding, e.g., JPEG2000 [43] and video coding, e.g., Motion JPEG2000, Motion-Compensated Embedded Zeroblock Coding (MC-EZBC) [16], schemes, leading to smooth adaptability within modern coding frameworks. Due to the multiresolution decomposition and the property to retain spatial synchronisation, which are not provided by other transforms (e.g., the Discrete Cosine Transform (DCT)), the Discrete Wavelet Transform (DWT) provides an ideal choice for image watermarking $[2,6-8,10,13-15,17,22,32,36,38-40,49,50]$ including algorithms developed for color image watermarking $[5,18,35,42]$. It is observed that the color watermarking algorithms often transform

Authors' addresses: Deepayan Bhowmik, deepayan.bhowmik@stir.ac.uk, University of Stirling, Stirling, FK9 4LA, United Kingdom; Charith Abhayaratne, c.abhayaratne@sheffield.ac.uk, University of Sheffield, Sheffield, S1 4ET, United Kingdom.

Permission to make digital or hard copies of all or part of this work for personal or classroom use is granted without fee provided that copies are not made or distributed for profit or commercial advantage and that copies bear this notice and the full citation on the first page. Copyrights for components of this work owned by others than ACM must be honored. Abstracting with credit is permitted. To copy otherwise, or republish, to post on servers or to redistribute to lists, requires prior specific permission and/or a fee. Request permissions from permissions@acm.org.

(C) 2019 Association for Computing Machinery.

$1551-6857 / 2019 / 0-$ ART0 $\$ 15.00$

https://doi.org/00.000/000.0000 
RGB to $\mathrm{YCbCr}$ color space and select $\mathrm{Y}$ component for watermark embedding (similar to gray scale watermarking). In wavelet-based image watermarking, different approaches have been used as follows:

- Choosing coefficients in a specific subband for embedding the watermark: e.g., embedding in high frequency subbands for better imperceptibility [9, 22, 28, 33, 42]; embedding in low frequency subband to achieve high robustness $[49,50]$ or the approaximation subband with the maximum variance $[6,8]$ and balancing imperceptibility and robustness with all subbands spread spectrum embedding [17, 38].

- Using different wavelet kernels: e.g., Haar or other Daubechies family orthogonal wavelets [5, 9, 10, 28, 33, 49] and biorthogonal wavelets [50].

- Optimising the host coefficient selection: e.g., choosing all coefficients in a subband [8, 9, 17]; using a threshold based on their magnitude significance [22]; the just noticeable difference(JND) [42, 50]; a mask based on the Human Visual System (HVS) model [6, 7, 9, 36]; a fusion rule-based mask for refining the selection of host coefficients [10] and blind re-quantization of a coefficient with respect to a group of coefficients within a given window $[28,33,38,49]$.

Though many independent algorithms are available in the literature, a gap, that requires a generalized mathematical analysis to identify the relationship between distortion performance and various wavelet-based watermarking parameters responsible for embedding distortion, was identified. To the best knowledge of the authors only handful of literature [23, 24] are available that attempted to address this issue, which, however, is limited to their own algorithms. We derive a model to establish the relationship between embedding distortion performance, in terms of mean square error (MSE) metric, and watermarking input parameters including wavelet kernels, subband selection and coefficient selection. Previous work [13] indicated that other objective metrics such as Structural Similarity Measure (SSIM) [46] or weighted PSNR (wPSNR) [34] capture watermark embedding distortion measurement similar to MSE/PSNR. Therefore, in this work we restrict ourselves to MSE to model the distortion as this is less complex and provides better insight in deriving the model. Such a model is useful to find the optimum input parameters, including, the wavelet kernel, coefficient selection and subband choices for wavelet domain image/video watermarking. The main contribution of this paper is to deriving a generalized model for distortion performance analysis of wavelet based watermarking algorithms. This is achieved by proposing

- Proposition 1: establishing the relationship between the noise power in the transform domain and the input signal domain.

- Proposition 2: deriving direct proportionality between the distortion performance metrics and the input parameters of a given wavelet based watermarking scheme for orthonormal wavelet bases, e.g., Haar, Daubechies-4, etc., which conserves energy in the signal domain as well as in the transform domain.

- Proposition 3: extending the above for non-orthonormal bases, including bi-orthogonal and non-linear wavelet kernels, to give a universal acceptance of the model.

The generalisation of our model is evaluated by fitting all major wavelet-based watermarking schemes into a common framework presented in [13]. Initial concepts and the results were reported earlier in the form of conference publications $[11,12]$ while this paper discusses the proposed scheme in detail, introduces non-linear kernels in the analysis and provides exhaustive performance evaluation. The scope of this work is strictly limited to embedding distortion analysis and excludes design and development of new robust watermarking algorithm that considers the derived model.

\section{WAVELETS}

Wavelet transforms represent a time domain signal in joint time-frequency domain. Various wavelet kernels, available in the literature including orthonormal Haar, Daubechies; biorthogonal 5/3, 9/7 [37] and non-linear wavelets $[1,3]$ with varying filter lengths and complexity, decompose the signal and represent signal singularities in different ways. In this subsection we revisited the set of wavelet kernels considered in this paper. 


\subsection{Orthonormal}

Orthonormal wavelet bases are one of most widely used and primary kernels that satisfies conditions for orthonormal basis and preserves energy between signal domain and transform domain. Traditionally a filter bank using convolution based approach is used to compute wavelet decomposition. This approach consists of two filter banks, one each for the analysis (forward transform) and the synthesis (inverse transform). During the analysis, the input signal is passed through two separate channels, using a high pass filter and a low pass filter followed by a down sampling operation by a factor of 2 , in each channel. To reconstruct the signal data, the transformed coefficients are first interpolated by an up sampling operation with a factor of 2 and then convolved with synthesis filter banks. Daubechies [19] proposed a set of orthonormal bases of compactly supported wavelet of varying filter length and coefficients. In this paper we have chosen four representative orthonormal wavelet kernels, i.e., Haar (HR), Daubechies length 4 (D4), 8 (D8) and 16 (D16) covering a representative range of shorter and longer filter length. The normalised coefficients for these filters are available from [19].

\subsection{Non-orthonormal}

Non-orthonormal wavelets are those where the associated wavelet transform is invertible but not necessarily orthonormal. This class includes bi-orthogonal and non-linear wavelets. Design of such wavelets usually allows more degrees of freedom and has been popular in many imaging applications, e.g., bi-orthogonal 5/3 and 9/7 wavelet kernels are part of JPEG2000 image compression standard [43].

2.2.1 Bi-orthogonal. We have chosen two representative bi-orthogonal wavelet kernels 5/3 and 9/7 and implemented using lifting based approach due to its lower complexity. The filters are realised by decomposing the signal into lifting steps by factoring its polyphase matrix using the Euclidean factoring algorithm [21]. Equations for these popular wavelet kernels can be obtained from [4].

2.2.2 Non-linear morphological wavelets. Non-linear wavelets are obtained by replacing the linear operations, such as weighted averaging, in lifting steps with non-linear operations. They can modify only the lifting step(s) affecting the low pass sub band (known as update step) [27], only the lifting step(s) affecting the high pass subbands (known as prediction step) [26] and the both types of lifting steps [3]. While orthonormal and biorthogonal wavelets are well discussed in the literature, non-linear wavelets are not so. As the analysis presented in this work calls upon understanding of vairous wavelet kernels, we briefly discuss the design steps for two morphological wavelet transforms used in this work: Morphological Haar (MH) and Median lifting on quincunx sampling (MQ).

Morphological Haar Since non-linearities are introduced in the scaling function, we design the Morphological Haar based on the 2D non-separable decomposition. We start with a 2D input signal $a^{0}$ and its four 2D polyphase components $(a, b, c, d)$, mapped by an invertible splitting operator $S^{2}$, i.e., $(a, b, c, d)=S^{2}\left(a^{0}\right)$. The operator $S^{2}$ forms a $\left(\begin{array}{ll}2 & 0 \\ 0 & 2\end{array}\right)$ sampling matrix and invertible mapping, called the lazy wavelet. The lazy wavelet is lifted to morphological 2-D Haar using $P_{3}, P_{2}, P_{1}, U$ lifting as follows to obtain output subbands, $a^{\prime}, b^{\prime}, c^{\prime}$ and $d^{\prime}$.

$$
\begin{aligned}
P_{3}: & d^{\prime}=\frac{1}{2}(d-(c+b-a)) . \\
P_{2}: & c^{\prime}=c-\left(a-d^{\prime}\right) . \\
P_{1}: & b^{\prime}=b-\left(a-d^{\prime}\right) . \\
U: & a^{\prime}=2\left[a+\operatorname{median}\left(0,\left(b^{\prime}-d^{\prime}\right),\left(c^{\prime}-d^{\prime}\right),\left(b^{\prime}+c^{\prime}\right)\right)\right] .
\end{aligned}
$$

The inverse transform is obtained by reversing the order of operation and the operator of the lifting steps $P_{3} P_{2} P_{1} U$. 

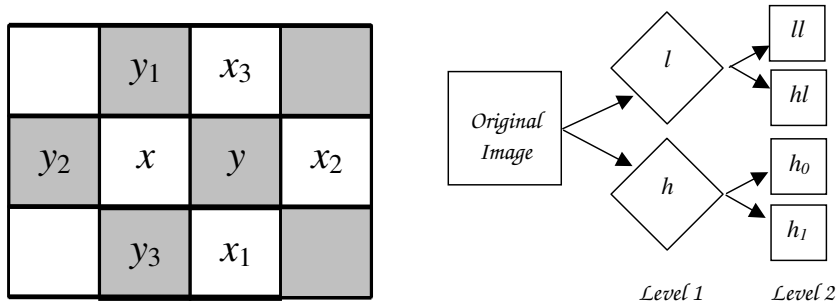

Fig. 1. Quincunx sampling: Left: Entries for prediction \& update lifting steps, Right: Subbands after two levels of decomposition.

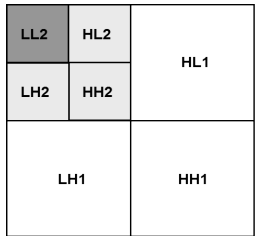

(a) DWT illustration

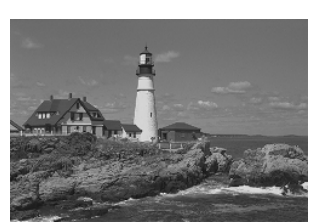

(b) LL1

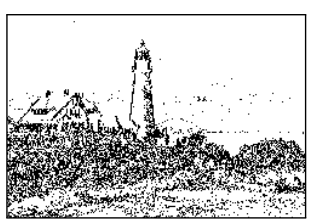

(c) LH1

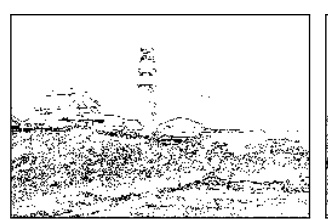

(d) HL1

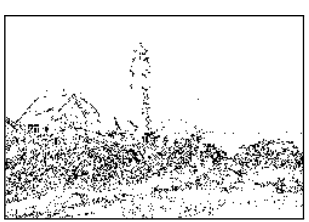

(e) $\mathrm{HH} 1$

Fig. 2. An example of multiresolution wavelet decomposition. (a) Illustration of two level DWT. (b)-(e) One level 2-D decomposition of an example image. (b), (c), (d) and (e) represent approximation (LL1), vertical (LH1), horizontal (HL1) and diagonal ( $\mathrm{HH} 1)$ subbands, respectively. Wavelet coefficients only with absolute values above the 0.9 quantile (largest $10 \%)$ are shown (as inverted image) for high frequency subbands ((c)-(e)) highlighting directional sensitivity.

Median lifting on quincunx sampling We design 2D non-separable wavelet transforms by using the quincunx sampling lattice with the corresponding sampling matrix $D=\left(\begin{array}{ll}1 & 1 \\ 1 & -1\end{array}\right)$. Its determinant is 2 and thereby results in two polyphase components of the 2D signal each having dimensions equal to $1 / \sqrt{2}$ of the original signal dimensions. We denote samples by $x$ and $y$ and we refer to their respective neighbours as $x_{1}, x_{2}, x_{3}$ and $y_{1}, y_{2}, y_{3}$ as shown in Fig. 1. The white and gray boxes represent samples from $x$ and $y$ polyphase components, respectively. In this paper we are concerned with lifting steps of the form:

$$
\begin{aligned}
\text { Prediction : } & y^{\prime}=y-\operatorname{median}\left(x, x_{1}, x_{2}, x_{3}\right), \\
\text { Update : } & x^{\prime}=x+\frac{1}{2} \operatorname{median}\left(y^{\prime}, y_{1}^{\prime}, y_{2}^{\prime}, y_{3}^{\prime}\right) .
\end{aligned}
$$

One level of decompositions results in two subimages whose dimensions are reduced by $\sqrt{2}$. The transform steps are repeated on the low pass subimage $(L)$. In order to comply with the four subband structure, the high pass subimage $(H)$ in every odd numbered decomposition level is further decomposed into two subimages whose dimensions are reduced by a factor 2 after two decompositions. Consequently, after every even numbered decomposition every image is decomposed into four subimages with three details and one approximation image. The right column of Fig. 1 shows the subimages after two levels of decompositions.

At this juncture, we define wavelet related acronyms used later in describing the proposed model. The 2D wavelet transform decomposes an image in frequency domain expressing coarse grain approximation (LL) of the original signal and three fine grain orientated edge information at multiple resolutions. LH, HL and HH subbands emphasise horizontal, vertical and diagonal contrasts within an image, respectively (refer Fig. 2), portraying prominent edges in various orientations. These notations are used herein to refer respective subbands. 


\section{WATERMARK EMBEDDING SCHEMES}

At this point, we describe the classical non-blind and blind categories of wavelet-based watermarking schemes that are used in Section 4 and Section 5 for embedding distortion analysis. The forward DWT (FDWT) is applied on the host image before watermark data is embedded within the selected subband coefficients. Once the watermark data was embedded, the inverse DWT (IDWT) concludes the watermarking process. Without loosing the generality, the embedding process can be expressed as

$$
C^{\prime}(m, n)=C(m, n)+\Delta(m, n)
$$

where $C^{\prime}(m, n)$ is the modified wavelet coefficient at $(m, n)$ position, $C(m, n)$ is the original value of the host coefficient and $\Delta(m, n)$ is the amount of modification due to watermark embedding. The extraction operation is performed after the FDWT. The extracted watermark is compared to the original embedded sequence before an authentication decision verifies the watermark presence. A wide variety of potential adversary attacks, including compression and filtering, can occur in an attempt to distort or remove any embedded watermark data.

The performance of the watermark embedding, i.e., embedding distortion is measured by comparing the watermarked image $\left(I^{\prime}\right)$ with the original unmarked image $(I)$ and is calculated by various metrics: 1) mean square error or peak signal to noise ratio (PSNR), 2) weighted PSNR (wPSNR) [34], 3) structural similarity measure (SSIM) [46], 4) just noticeable difference (JND) [47] and 5) subjective quality measurement [31]. Among these the first is widely used due to it simplicity and low computation complexity. However, experiments suggest that for most host images, if the PSNR is greater than 35dB, other objective measures, such as wPSNR and SSIM, are highly correlated with the PSNR / MSE values [13]. Therefore in this work, we chose MSE as the distortion measurement metric and derived relationships proposed in Section 4 and Section 5.

\subsection{Non-blind Watermarking}

Magnitude-based multiplicative watermarking $[7-9,15,25,30,41,48]$ is a popular choice when using a non-blind watermarking system, due to its simplicity. Wavelet coefficients are modified based on the watermark strength parameter, $\alpha$, the magnitude of the original coefficient, $C(m, n)$ and the watermark information, $W(m, n)$. The watermarked coefficients, $C^{\prime}(m, n)$, are obtained as follows:

$$
C^{\prime}(m, n)=C(m, n)+\alpha W(m, n) C(m, n)=C(m, n)(1+\alpha W(m, n)) .
$$

$W(m, n)$ is derived from a pseudo-random binary sequence, $b$, using weighting parameters, $W_{1}$ and $W_{2}$ (where $\left.W_{2}>W_{1}\right)$, which are assigned as follows:

$$
W(m, n)= \begin{cases}W_{2} & \text { if } b=1 \\ W_{1} & \text { if } b=0 .\end{cases}
$$

To obtain the extracted watermark, $W^{\prime}(m, n)$, Eq. (8) is rearranged as:

$$
W^{\prime}(m, n)=\frac{C^{\prime}(m, n)-C(m, n)}{\alpha C(m, n)} .
$$

Since the non-watermarked coefficients, $C(m, n)$, are needed for comparison, this results in non-blind extraction. A threshold limit of $T_{w}=\frac{W_{1}+W_{2}}{2}$ is used to determine the extracted binary watermark $b^{\prime}$ as follows:

$$
b^{\prime}= \begin{cases}1 & \text { if } W^{\prime}(m, n) \geq T_{w} \\ 0 & \text { if } W^{\prime}(m, n)<T_{w}\end{cases}
$$




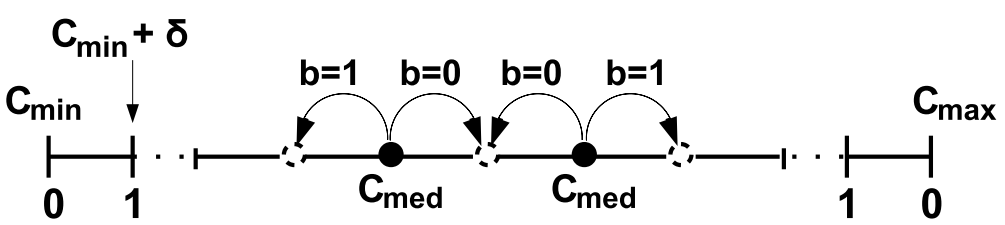

Fig. 3. Blind quantisation-based coefficient embedding.

\subsection{Blind Watermarking}

Quantization-based watermarking [28, 29, 33, 38, 44, 49] is a blind scheme which relies on modifying various coefficients towards a specific quantization step. As proposed in [49], the algorithm is based on modifying the median coefficient towards the step size, $\delta$, by using a running non-overlapping $3 \times 1$ window. The altered coefficient must retain the median value of the three coefficients within the window, after the modification. The equation calculating $\delta$ is described as follows:

$$
\delta=\alpha \frac{\left(C_{\min }\right)+\left(C_{\max }\right)}{2},
$$

where $C_{\min }$ and $C_{\max }$ are the minimum and maximum coefficients, respectively. The median coefficient, $C_{\text {med }}$, is quantised towards the nearest step, depending on the binary watermark, $b$. Quantisation-based watermark embedding is shown in Fig. 3 . The extracted watermark, $b^{\prime}$, for a given window position, is extracted by

$$
b^{\prime}=\left[\frac{C_{\text {max }}-C_{\text {med }}}{\delta}\right] \% 2,
$$

where \% denotes the modulo operator to detect an odd or even number and $C_{m e d}$ is the median coefficient value within the $3 \times 1$ window.

\subsection{Authentication of extracted watermarks}

Authentication is performed by comparison of the extracted watermark with the original watermark information and computing closeness between the two in a vector space. Common authentication methods are defined by calculating the similarity correlation or Hamming distance, $H$, between the original embedded and extracted watermark as follows:

$$
H\left(b, b^{\prime}\right)=\frac{1}{N} \sum b \oplus b^{\prime},
$$

where $N$ represents the length of the watermark sequence and $\oplus$ is the XOR logical operation between the respective bits.

\section{EMBEDDING DISTORTION ANALYSIS FOR ORTHONORMAL BASES}

\subsection{Preliminaries}

The embedding distortion performance is measured by MSE, which can be defined as follows:

Definition 1. The Mean Square Error (MSE) or average noise power in pixel domain between original image I and watermarked image $I^{\prime}$ is defined by:

$$
M S E=\frac{1}{N \times M} \sum_{m=0}^{N-1} \sum_{n=0}^{M-1}\left(I(m, n)-I^{\prime}(m, n)\right)^{2},
$$

where $M$ and $N$ are the image dimension and $m$ and $n$ indicate each pixel position. 
For simplicity, during the derivation of the model often we shall refer $1 \mathrm{D}$ signals which is then inferred to $2 \mathrm{D}$ image signals in the following text. In order to formulate the model we show the transformation of noise energy from frequency domain to the signal domain using Parseval's equality. In Parseval's equality, the energy is conserved between an input signal and the transform domain coefficient in the case of an orthonormal filter bank wavelet base [45]. Assuming the input signal $x[n]$ with the length of $n \in \mathcal{Z}$ and the corresponding transformed domain coefficients of $y[k]$ where $k \in \mathcal{Z}$, according to energy conservation theorem,

$$
\|x\|^{2}=\|y\|^{2} \text {. }
$$

\subsection{The model}

Proposition 1. Sum of the noise power in the transform domain is equal to sum of the noise power in the input signal for orthonormal transforms. If the input signal noise is defined by $\Delta x[n]$ and the noise in transform domain is $\Delta y[k]$ then,

$$
\sum_{n}|\Delta x[n]|^{2}=\sum_{k}|\Delta y[k]|^{2},
$$

where $n \in \mathcal{Z}$ is the length of the input signal and $k \in \mathcal{Z}$ is the length in the transform domain, respectively.

Proof. Assuming $\Delta y$ is the noise introduced in wavelet domain and $\Delta x$ is the modified signal after the inverse transform, we can define the relationship between the noise in the wavelet coefficient and the noise in the modified signal using the following equations.

$$
\left(\begin{array}{l}
x_{e}(z)+\Delta x_{e}(z) \\
x_{o}(z)+\Delta x_{o}(z)
\end{array}\right)=\left(\begin{array}{ll}
h_{e}^{\prime}(z) & h_{o}^{\prime}(z) \\
g_{e}^{\prime}(z) & g_{o}^{\prime}(z)
\end{array}\right)\left(\begin{array}{l}
y_{e}(z)+\Delta y_{e}(z) \\
y_{o}(z)+\Delta y_{o}(z)
\end{array}\right) .
$$

where $h^{\prime}(z)$ represents the low pass filter coefficients and $g^{\prime}(z)$ is the high pass filter coefficients and the subscripts $e$ and $o$ denote even and odd indexed terms, respectively.

Using the Linearity property of the $Z$-transform of the filter coefficients and signals in the polyphase matrix one can write the polyphase matrix form of the noise in the output signal:

$$
\left(\begin{array}{l}
\Delta x_{e}(z) \\
\Delta x_{o}(z)
\end{array}\right)=\left(\begin{array}{ll}
h_{e}^{\prime}(z) & h_{o}^{\prime}(z) \\
g_{e}^{\prime}(z) & g_{o}^{\prime}(z)
\end{array}\right)\left(\begin{array}{l}
\Delta y_{e}(z) \\
\Delta y_{o}(z)
\end{array}\right) .
$$

Recalling the energy conservation in Eq. (16) and as stated in Eq. (19) we can conclude that

$$
\begin{aligned}
\sum\left|\Delta x_{e}\right|^{2}+\sum_{n}\left|\Delta x_{o}\right|^{2} & =\sum_{n}\left|\Delta y_{e}\right|^{2}+\sum\left|\Delta y_{o}\right|^{2}, \\
\sum_{k}|\Delta x[n]|^{2} & =\sum_{k}|\Delta y[k]|^{2} .
\end{aligned}
$$

This proves Proposition 1 (detailed derivation of this is proof can be found in Appendix A).

Using the generalized framework, the Eq. (20) can be applied to build the relationship between the modification energy in the coefficient domain to embed the watermark and the distortion performance metrics. In this model we made propositions for two different categories of embedding schemes, discussed in Proposition 2.

Proposition 2. In a wavelet based watermarking scheme, mean square error (MSE) of the watermarked image is directly proportional to the sum of energy of the modification values of selected wavelet coefficients. The modification value itself is a function of the coefficients and therefore we propose two different cases based on the categorization. Case A: Non blind model. For the magnitude alteration based embedding method (non blind algorithm), the modification is a function of the selected coefficient to be watermarked and the relationship between (MSE) and the selected coefficient $\left(C_{m, n}\right)$ is expressed as:

$$
M S E \propto \sum\left|f\left(C_{m, n}\right)\right|^{2} .
$$


Case B. Blind model. For the re-quantization based method (blind algorithm), the modification is a function of the neighboring wavelet coefficients of the selected median coefficient to be watermarked and the relationship between MSE and the wavelet coefficients $C_{\text {min }}$ and $C_{\max }$ is expressed as:

$$
M S E \propto \sum\left|f\left(C_{\text {min }}, C_{\text {max }}\right)\right|^{2} .
$$

PRoof. In a wavelet based watermark embedding scheme the watermark information is inserted by modifying the wavelet coefficients. This watermark insertion can be considered as introducing noise in the transform domain. Hence the sum of the energy of the modification value due to watermark embedding in the wavelet domain is equal to the sum of the noise energy in the transform domain as stated in Proposition 1. From Eq. (7) and Eq. (17), the energy sum of the modification value $\Delta_{k}$ can be defined as:

$$
\sum_{k}\left|\Delta_{k}\right|^{2}=\sum_{k}|\Delta y[k]|^{2} .
$$

Similarly, the pixel domain distortion performance metrics which is represented by MSE is considered as the noise error created in the signal due to the noise in wavelet domain. Therefore, the sum of the noise energy in the input signal is equal to the sum of the noise error energy MSE in the pixel domain:

$$
\text { MSE }=\sum_{n}|\Delta x[n]|^{2} .
$$

Now the relationship between the distortion performance metrics MSE of the watermarked image and the coefficient modification value which is normally a function of the selected wavelet coefficients can be decided using the Proposition 1. Thus from Eq. (23) and Eq. (24) we can write:

$$
\text { MSE. }(M \times N)=\sum_{m, n}\left|\Delta_{m, n}\right|^{2},
$$

where $M$ and $N$ are the image dimensions. Hence for any watermarked image, the average noise power MSE is proportional to the sum of the energy of the modification values of the selected wavelet coefficients:

$$
\operatorname{MSE} \propto \sum_{m, n}\left|\Delta_{m, n}\right|^{2} .
$$

Now with the help of the categorization in the generalized form of the popular wavelet based watermarking schemes as discussed in Section 3, a relationship is established between the error energy of the watermarked image and the selected wavelet coefficient energy of the host image. For a magnitude alteration based algorithm, which is a category of non blind watermarking algorithm, the mean square error MSE is directly proportional to the sum of the energy of the modification value $\Delta$ which is a function of wavelet coefficient value as stated below:

$$
\operatorname{MSE} \propto \sum\left|f\left(C_{m, n}\right)\right|^{2} .
$$

Similarly for the re-quantization based method (blind watermarking) the mean square error depends on the neighboring wavelet coefficient values. In this case the modification energy $\left|\Delta_{m, n}\right|^{2}$ hold an inequality due the modification range $-\delta \leq \Delta_{m, n} \leq \delta$ :

$$
\left|\Delta_{m, n}\right|^{2} \leq|\delta|^{2} .
$$

Therefore the upper bound of the mean square error MSE is defined by:

$$
\text { MSE } \propto \sum\left|f\left(C_{\text {min }}, C_{\text {max }}\right)\right|^{2} .
$$

This proves Proposition 2. 
4.2.1 An example of non blind model. Considering a specific case of the non blind algorithm in [30] the modification value $\Delta$ is a direct function of wavelet coefficient $\left(\Delta_{m, n}=\alpha C_{m, n} W_{m, n}\right)$. Hence Eq. (27) can be modified and the MSE can be expressed as:

$$
\operatorname{MSE} \propto \sum_{k=1}^{l}|C(k)|^{2},
$$

where $C(k)$ is the selected coefficients to be watermarked and $l$ is the number of such selected coefficients.

4.2.2 An example of blind embedding model. In an blind embedding algorithm suggested in [49], the quantization step $\delta$ is defined as:

$$
\delta=\gamma \frac{C_{\max }+C_{\min }}{2},
$$

where $\gamma$ is the user defined watermark weighting factor. As the modification value $\Delta$ depends on $\delta$, with reference to Eq. (29), the relationship between the maximum limit of MSE and wavelet energy is defined by the following equation:

$$
\operatorname{MSE} \propto \sum_{k}\left(C(k)_{\text {max }}+C(k)_{\text {min }}\right)^{2},
$$

where $C(k)_{\max }$ and $C(k)_{\min }$ are the neighborhood coefficients of the median value and $k$ is the number of such selected median value.

\section{EMBEDDING DISTORTION ANALYSIS FOR NON-ORTHONORMAL WAVELET BASES}

\subsection{Preliminaries}

Recalling Parseval's Equality, Eq. (16) is true for orthonormal transforms where energy is conserved between transforms. On the contrary, non-orthonormal wavelets such as biorthogonal wavelets do not hold conservation of energy. However for a stable expansion, the transform domain coefficients have to satisfy the Eq. (33) [45].

$$
A \sum_{k}|y[k]|^{2} \leq\|x\|^{2} \leq B \sum_{k}|y[k]|^{2},
$$

where $A$ and $B$ are the orthonormality correction factor. A detailed derivation of this is available from [20].

\subsection{The model}

Based on the discussed propositions and the definitions we shall build the extended model and make the new propositions. As suggested in Eq. (33), for a non-orthonormal wavelet base an orthonormality correction factor is required and we shall call this as a weighting parameter $W_{t}$ which is defined as follows:

$$
W_{t}=\frac{\|x\|^{2}}{\sum_{k}|y[k]|^{2}},
$$

where $x$ and $y$ is the input signal and the transform domain coefficients, respectively.

Therefore at this point we can extend Proposition 1 to a more generalized form. In a polyphase decomposition we use different low pass and high pass filter banks. Hence at each of the different transform points, we receive different weighting parameters $W_{t}^{g}$ and $W_{t}^{h}$, corresponding to high or low pass filters, respectively. Now the Proposition 1 can be extended as follows, accommodating the weighting parameter for non-orthonormal transforms:

$$
\begin{aligned}
\sum\left(\left|\Delta x_{e}\right|^{2}+\left|\Delta x_{o}\right|^{2}\right) & =W_{t}^{g} \sum\left(\left|\Delta y_{e}\right|^{2}+\left|\Delta y_{o}\right|^{2}\right)+W_{t}^{h} \sum\left(\left|\Delta y_{e}\right|^{2}+\left|\Delta y_{o}\right|^{2}\right), \\
\sum_{n}|\Delta x[n]|^{2} & =W_{t}^{g} \sum\left(\left|\Delta y_{e}\right|^{2}+\left|\Delta y_{o}\right|^{2}\right)+W_{t}^{h} \sum\left(\left|\Delta y_{e}\right|^{2}+\left|\Delta y_{o}\right|^{2}\right) .
\end{aligned}
$$


Now using the generalized framework, Eq. (35) can be applied to build the relationship between the modification energy in the coefficient domain to embed the watermark and the distortion performance metrics for orthonormal as well as non-orthonormal wavelet bases.

PROPOSITION 3. In a wavelet based watermarking scheme, the mean square error (MSE) of the watermarked image is directly proportional to the weighted sum of the energy of the modification values of the selected wavelet coefficients.

$$
\left.M S E \propto W_{t}^{\Theta r} \sum\left|\Delta_{m, n}\right|\right)\left.\right|^{2},
$$

where $W_{t}$ is the weighting parameter at each subband and $\Theta$ represents the subband number at $\Upsilon$ decomposition level.

Proof. In order to prove this proposition, we recall Eq. (23) and Eq. (24) to combine them with Eq. (35) and the combined form can be written as:

$$
\begin{aligned}
\operatorname{MSE}(N \times M) & =\sum_{n}|\Delta x[n]|^{2}, \\
& =W_{t}^{g} \sum_{n}|\Delta y[n]|^{2}+W_{t}^{h} \sum_{n}|\Delta y[n]|^{2}, \\
& =W_{t}^{g} \sum_{m, n}\left|\Delta_{m, n}\right|^{2}+W_{t}^{h} \sum_{m, n}\left|\Delta_{m, n}\right|^{2} .
\end{aligned}
$$

Hence for any watermarked image, the average noise power MSE is proportional to the sum of the weighted energy of the modification values of the selected wavelet coefficients:

$$
\operatorname{MSE} \propto W_{t}^{g} \sum_{m, n}\left|\Delta_{m, n}\right|^{2}+W_{t}^{h} \sum_{m, n}\left|\Delta_{m, n}\right|^{2} .
$$

Now in the case of 2-D wavelet decompositions, the wavelet kernel transfer function, for each subband at each decomposition level are different and so that the weighting parameters are. Hence the $\Delta$ in Eq. (38) are associated with a corresponding weighting parameter for each subband at each decomposition level. We define the weighting parameter as $W_{t}^{\Theta \Upsilon}$ at each subband and $\Theta$ represents the subband number at $\Upsilon$ decomposition level and therefore Eq. (38) can be re-written as:

$$
\left.\operatorname{MSE} \propto W_{t}^{\Theta \Upsilon} \sum\left|\Delta_{m, n}\right|\right)\left.\right|^{2} .
$$

This proves Proposition 3.

Therefore, using Eq. (39), the Eq. (21) and Eq. (22) can be extended for non blind and blind model to Eq. (40) and Eq. (41), respectively, as follows:

$$
\begin{aligned}
& \text { MSE } \propto \sum W_{t}^{\Theta \Upsilon}\left|f\left(C_{m, n}\right)\right|^{2} . \\
& \operatorname{MSE} \propto \sum W_{t}^{\Theta \Upsilon}\left|f\left(C_{\text {min }}, C_{\text {max }}\right)\right|^{2} .
\end{aligned}
$$

Hence the above equation can universally used for various wavelet kernels, where for orthonormal wavelet kernels the value of the weighting parameters are equal to unity. For non-orthonormal wavelet kernel, different weighting parameter values are suggested in next section for different subbands at each decomposition level. 


\begin{tabular}{c|c|c|c|c}
\hline & $9 / 7$ & $5 / 3$ & $\mathrm{MH}$ & $\mathrm{MQ}$ \\
\hline LL3 & $1.00 \pm 0.00$ & $0.98 \pm 0.00$ & $1.00 \pm 0.00$ & $0.98 \pm 0.01$ \\
LH3 & $1.37 \pm 0.08$ & $0.57 \pm 0.03$ & $1.00 \pm 0.00$ & $0.15 \pm 0.01$ \\
HL3 & $1.13 \pm 0.02$ & $0.57 \pm 0.03$ & $1.00 \pm 0.00$ & $0.17 \pm 0.00$ \\
HH3 & $1.31 \pm 0.06$ & $0.53 \pm 0.02$ & $1.00 \pm 0.00$ & $0.12 \pm 0.00$ \\
\hline LL2 & $1.00 \pm 0.00$ & $0.99 \pm 0.00$ & $1.00 \pm 0.00$ & $0.98 \pm 0.00$ \\
LH2 & $1.22 \pm 0.06$ & $0.69 \pm 0.03$ & $1.00 \pm 0.00$ & $0.31 \pm 0.01$ \\
HL2 & $1.07 \pm 0.03$ & $0.74 \pm 0.04$ & $1.00 \pm 0.00$ & $0.52 \pm 0.00$ \\
HH2 & $1.17 \pm 0.05$ & $0.81 \pm 0.03$ & $1.01 \pm 0.00$ & $0.41 \pm 0.01$ \\
\hline LL1 & $1.00 \pm 0.00$ & $0.99 \pm 0.00$ & $1.00 \pm 0.00$ & $0.99 \pm 0.00$ \\
LH1 & $1.22 \pm 0.04$ & $1.31 \pm 0.03$ & $1.00 \pm 0.00$ & $0.94 \pm 0.02$ \\
HL1 & $1.09 \pm 0.02$ & $1.31 \pm 0.03$ & $1.00 \pm 0.00$ & $1.97 \pm 0.03$ \\
HH1 & $1.34 \pm 0.04$ & $2.43 \pm 0.08$ & $1.02 \pm 0.00$ & $1.64 \pm 0.05$ \\
\hline
\end{tabular}

Table 1. Weighting parameter values of each subband at each decomposition level for various non-orthonormal wavelets.

\subsection{Calculation of the weighting parameters}

The weighting parameters are calculated for each subband at each decomposition level for various wavelet kernels. We have done a three level decomposition and calculated the weighting parameter value for each of the ten subbands. A set of different non-orthonormal wavelet kernels including bi-orthogonal 5/3 and 9/7, are chosen for the experimental simulations. Although the propositions made here assumed Linearity property of wavelet kernels, we have experimentally simulated and observe the similar proposition on non-linear wavelets, such as, Morphological Haar and Quincunx domain Morphological wavelets (described in Section 2.2.2). While calculating the weighting parameters, we have considered the energy ratio for each subband one at a time while keeping other subband values to zero in Eq. (42).

$$
W_{t}^{\Theta \Upsilon}=\frac{\|x\|^{2}}{\sum\left|y^{\Theta \Upsilon}\right|^{2}},
$$

where $W_{t}^{\Theta \Upsilon}$ is the weighting parameter at $\Theta$ subband at $\Upsilon$ decomposition level, $y^{\Theta \Upsilon}$ is the coefficient value at $\Theta$ subband at $\Upsilon$ decomposition level and $x$ is the output pixel values after the inverse wavelet transform. The weighting parameters are calculated for the experimental image set and generalized by averaging them. It is observed that these parameters are image independent. The corresponding weighting parameters for different subbands at each decomposition levels are calculated and shown TABLE 1 along with the error. The errors presented here display accuracy up to the $95 \%$ confidence interval.

\section{EXPERIMENTAL RESULTS AND DISCUSSION}

This section describes the extensive experimental results and analysis in support of proposed distortion analysis models. Firstly we provided details of experimental setup and parameters followed by results and discussions for orthonormal and non-orthonormal cases, respectively.

It is to be noted that the scope of this work is strictly limited to embedding distortion analysis. Design, development of new watermarking algorithms that consider the derived model and associated robustness results are outside the scope of this paper and are planned as future work. Additionally, to our best knowledge of the authors embedding distortion analysis proposed in this work is unique and due to lack of any other comparable 
0:12 Bhowmik and Abhyaratne, et al.

\begin{tabular}{c|c|c|c|c|c|c|c|c}
\hline & \multicolumn{3}{|c|}{ Non blind model } & \multicolumn{4}{c}{ Blind model } \\
\cline { 2 - 9 } & HR & D4 & D8 & D16 & HR & D4 & D8 & D16 \\
\hline LL3 & 0.81 & 0.81 & 0.81 & 0.81 & 0.66 & 0.68 & 0.68 & 0.73 \\
LH3 & 0.93 & 0.94 & 0.96 & 0.97 & 0.78 & 0.68 & 0.61 & 0.58 \\
HL3 & 0.98 & 0.99 & 0.99 & 0.99 & 0.78 & 0.92 & 0.94 & 0.97 \\
HH3 & 0.96 & 0.97 & 0.98 & 0.98 & 0.82 & 0.81 & 0.73 & 0.72 \\
\hline LH2 & 0.98 & 0.98 & 0.99 & 0.99 & 0.80 & 0.82 & 0.75 & 0.81 \\
HL2 & 0.99 & 0.99 & 0.99 & 0.99 & 0.92 & 0.92 & 0.94 & 0.97 \\
HH2 & 0.99 & 0.99 & 0.99 & 0.99 & 0.83 & 0.80 & 0.85 & 0.89 \\
\hline LH1 & 0.99 & 0.99 & 0.99 & 0.99 & 0.89 & 0.90 & 0.89 & 0.90 \\
HL1 & 0.99 & 0.99 & 0.99 & 0.99 & 0.84 & 0.90 & 0.96 & 0.94 \\
HH1 & 0.99 & 0.99 & 0.99 & 0.99 & 0.90 & 0.91 & 0.93 & 0.96 \\
\hline
\end{tabular}

Table 2. Correlation coefficient values between sum of energy and the MSE for different wavelet kernel in various subbands.

study, it is not possible to compare our experimental outcome with other works. Existing works generally measure the distortion to evaluate the performance of their methods. In that context we consider the results without the proposed model as the base line and show improvements when the model was applied.

\subsection{Experimental setup}

In order to obtain consistent results, a set of 20 images including standard test images and images from the Kodak image test set ${ }^{1}$ along with a $64 \times 64$ binary logo were used in all experiments. Our previous experiments suggest that type of logo has negligible impact on the watermarking distortion [13] and therefore choice of a logo has no impact in the proposed work. The sum of the energy of the selected wavelet coefficients and the MSE of the watermarked image have been calculated for the test images with a combination of different input parameters. As the wavelet coefficients varies greatly in different subbands we have considered the performances of all subbands separately after a 3-level wavelet decomposition. After three level of wavelet decompositions, ten subbands are created, such as, LL3, HL3, LH3 and HH3 at 3rd decomposition level, HL2, LH2 and HH2 at 2nd decomposition level and HL1, LH1 and HH1 at 1st decomposition level. Also a set of different wavelet kernels having various filter lengths are selected to perform the simulations. In order to verify our proposed model, we have simulated and studied the performance of different wavelet kernels such as Haar (HR), Daubechies-4 (D4), Daubechies-8 (D8) and Daubechies-16 (D16) for orthonormal and bi-orthogonal 9/7, 5/3 and non-linear Morphological Haar (MH) and Quincunx domain Morphological wavelets (MQ) for non-orthonomal models, respectively. Two different sets of results are obtained for each non blind and blind model, and displayed to verify the effects of different input parameters which are responsible for embedding distortion performance. These two sets of experimental arrangements and resulting plots are discussed separately in the subsections below.

\subsection{Orthonormal cases}

The simulations of the proposed embedding distortion model for orthonormal wavelet kernels are performed using the experimental set up above. We have used the test image set, with three level wavelet decomposition. We

\footnotetext{
${ }^{1}$ http://r0k.us/graphics/kodak/
} 


\begin{tabular}{c|c|c|c|c|c|c|c|c}
\hline & \multicolumn{3}{|c|}{ Non blind model } & \multicolumn{4}{c}{ Blind model } \\
\cline { 2 - 9 } & $9 / 7$ & $5 / 3$ & MH & MQ & $9 / 7$ & $5 / 3$ & MH & MQ \\
\hline LL3 & 0.80 & 0.81 & 0.81 & 0.81 & 0.77 & 0.82 & 0.43 & 0.78 \\
LH3 & 0.95 & 0.90 & 0.93 & 0.97 & 0.78 & 0.51 & 0.73 & 0.86 \\
HL3 & 0.99 & 0.97 & 0.98 & 0.95 & 0.96 & 0.94 & 0.73 & 0.92 \\
HH3 & 0.95 & 0.94 & 0.95 & 0.96 & 0.80 & 0.84 & 0.69 & 0.83 \\
\hline LH2 & 0.97 & 0.97 & 0.98 & 0.99 & 0.81 & 0.81 & 0.70 & 0.94 \\
HL2 & 0.99 & 0.99 & 0.99 & 0.99 & 0.96 & 0.97 & 0.90 & 0.86 \\
HH2 & 0.99 & 0.99 & 0.99 & 0.98 & 0.89 & 0.88 & 0.84 & 0.93 \\
\hline LH1 & 0.99 & 0.99 & 0.99 & 0.97 & 0.88 & 0.87 & 0.90 & 0.89 \\
HL1 & 0.97 & 0.97 & 0.98 & 0.99 & 0.75 & 0.91 & 0.91 & 0.95 \\
HH1 & 0.99 & 0.99 & 0.99 & 0.99 & 0.95 & 0.89 & 0.88 & 0.94 \\
\hline
\end{tabular}

Table 3. Correlation coefficient values between sum of energy and the MSE for different wavelet kernel in various subbands.

have simulated and studied the performance of four different wavelet kernels such as Haar (HR), Daubechies-4 (D4), Daubechies-8 (D8) and Daubechies-16 (D16). Details of non blind and blind models are described below.

6.2.1 Non blind model. In experiment Set 1, we have considered the non blind type watermark embedding model as described in Section 4.2.1. The sum of energy of the selected wavelet coefficients to be modified and MSE of the watermarked image have been calculated using $\alpha=0.5$ and the binary watermark logo for each selected method. The logo was repeatedly embedded on all coefficients in a selected subband making sure we measure the embedding noise uniformly. We have used various wavelet kernels and observed the results for each selected subbands. The correlation coefficients are also calculated and presented in TABLE 2.

In another representation a set of graphs are plotted in Fig. 4(a) to present the average values of the MSE and the sum of energy for the test image set for four different wavelet kernels. The error bars denote the accuracy up to the $95 \%$ confidence interval. For display purposes the sum of energy values were scaled, so that they can be shown on the same plot for comparing the trend.

In the experiment Set 2, the performance for ten different subbands are plotted for each wavelet kernel in a similar fashion as mentioned in experiment Set 1 in order to observe the trend. The results are shown in Fig. 5(a). As earlier, a 95\% confidence interval is considered which is denoted by the error bars and the LL3 values are scaled suitably in all cases to observe the trends.

6.2.2 Blind model. We have conducted experimental simulations for the blind model as described in Section 4.2.2. A similar set of experimental set up is followed as in non blind model with $\gamma=0.04$ and 0.2 for LL3 subband and other high frequency subbands, respectively. The correlation coefficients, average pattern graphs for various wavelet kernels and ten different subbands are presented in TABLE 2, Fig. 4(a) and Fig. 5(b), respectively.

The simulation results show a strong correlation between MSE of the watermarked image and the energy sum of the selected wavelet coefficients to be modified. It is observed that for the non blind model, the correlation coefficient value is more than 0.80 and more than 0.66 in the case of blind model, for different wavelet kernels and various selected subbands. On the other hand, a similar graph patterns are observed in Fig. 4 and Fig. 5, which show the proportionality trend between MSE and the energy sum as proposed in the model. Lower correlation coefficients are observed for blind model due to the reason that the proportionality relationship only defines the upper bound in Eq. (29) and Eq. (32). 

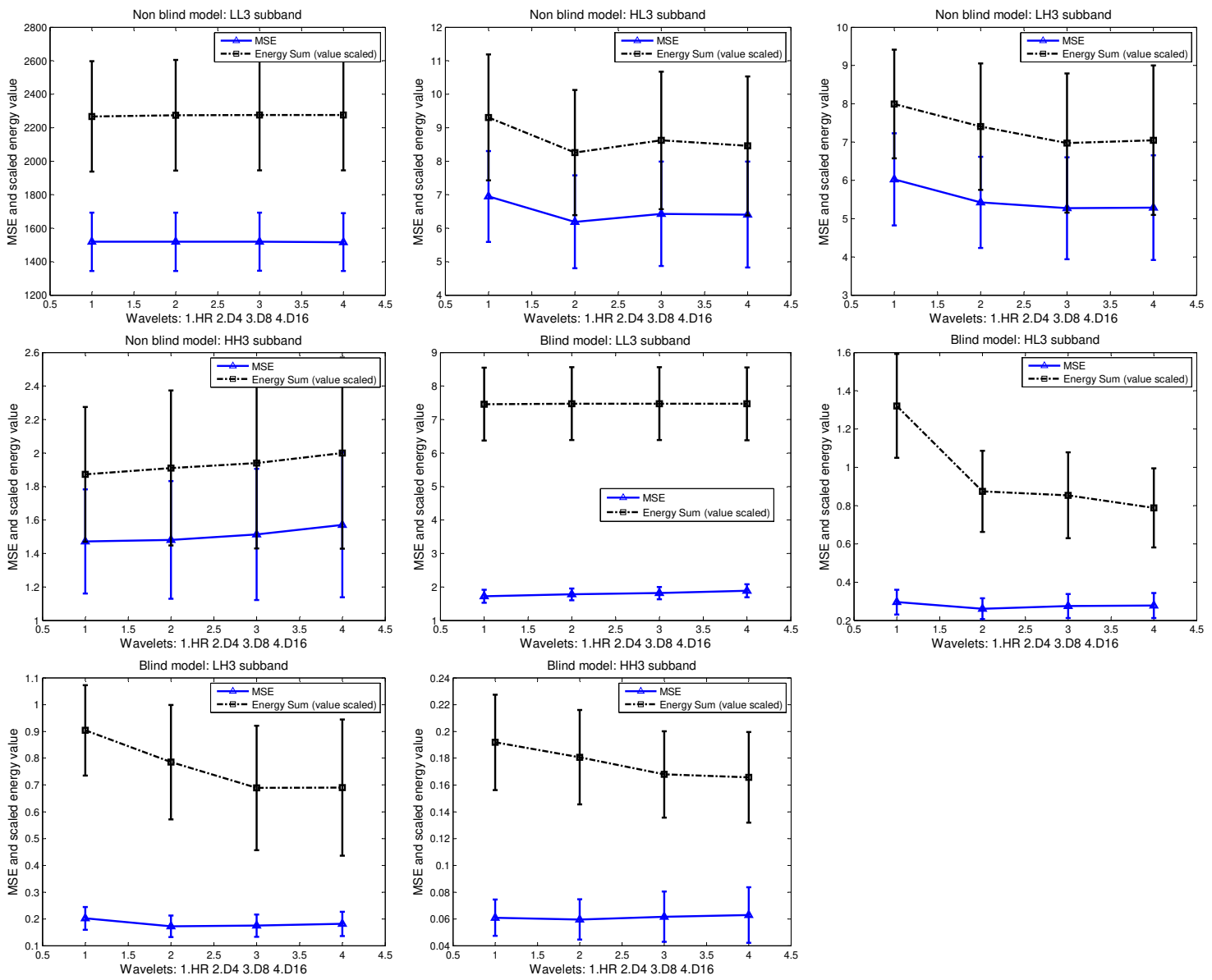

Fig. 4. Watermark embedding performance graph for different subbands. Four different wavelet kernels are compared: 1. HR, 2. D4, 3. D8 and 4. D16. Both non-blind and blind methods are shown in first four and in last four figures, respectively.

\subsection{Non-orthonomral cases}

The simulations of the proposed embedding distortion model for non-orthonormal wavelet kernels are performed using a similar set up as used in Section 6.2 for orthonormal wavelets. We have used the same test image set, with three level wavelet decomposition. Four different non-orthonormal wavelet kernels, namely, bi-orthogonal 9/7 and 5/3 and non-linear Morphological Haar (MH) and Quincunx domain Morphological wavelets (MQ), are simulated and studied here. For each simulations, first, results are shown without considering the weighting parameters $\left(W_{t}^{\Theta \Upsilon}\right)$ and then the corresponding results using weighting parameters from TABLE 1.

6.3.1 Non blind model. The experimental simulations for non blind model as described in Eq. (40) is performed and the correlation coefficients are calculated and represented in TABLE 3. The average values of the MSE and the sum of energy are shown in Fig. 6. Row 1 and row 2 represent the results without and with considering the weighting parameter, while calculating the energy sum, respectively. The error bars denote the accuracy up to 

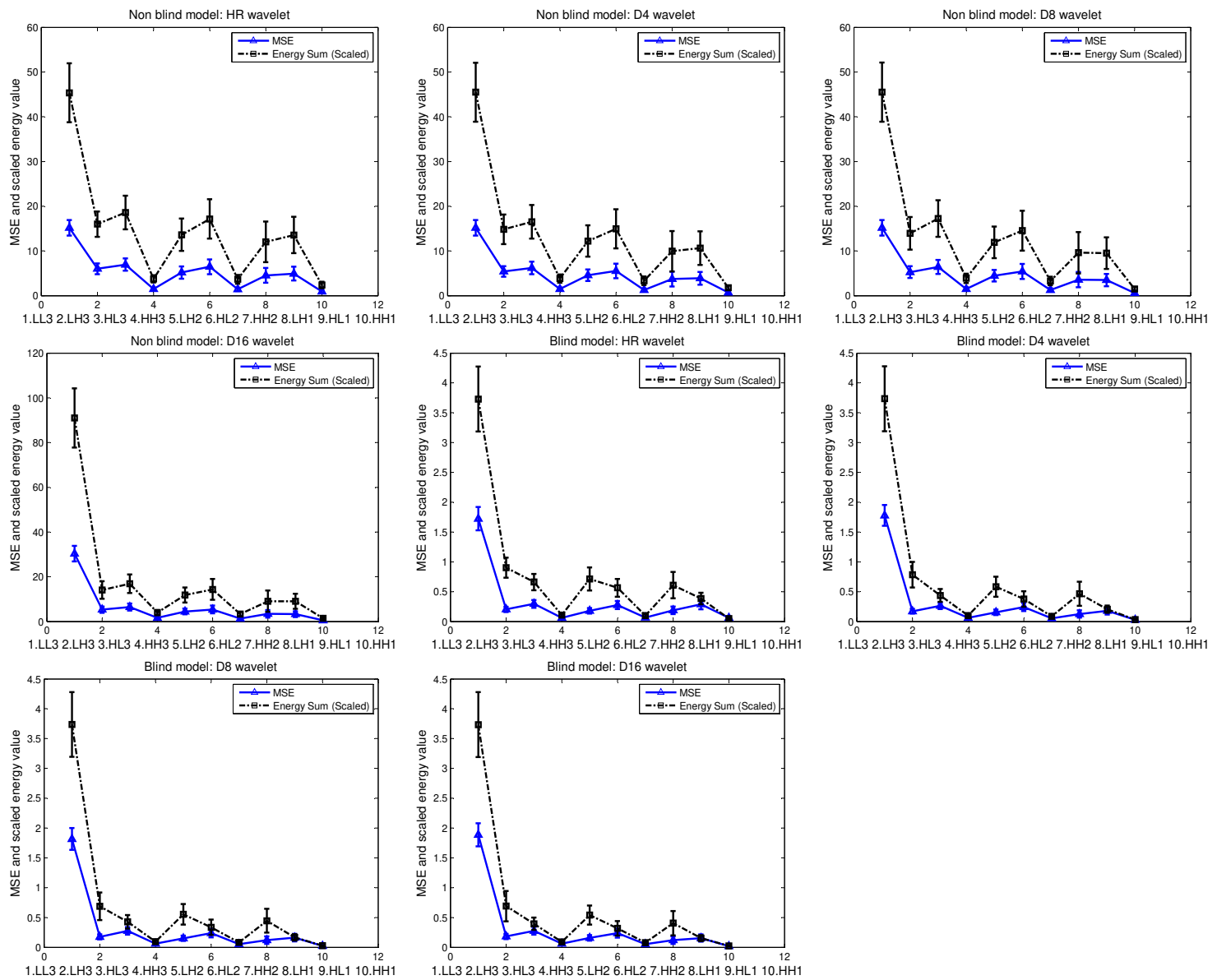

Fig. 5. Watermark embedding performance graph for various wavelets in different subband. Both non-blind and blind methods are shown in first four and in last four figures, respectively.

the $95 \%$ confidence interval. For display purposes the sum of energy value was scaled, so that they can be shown on the same plot for comparing the trend.

In the other experiment set the subbands are compared and the results are shown in Fig. 7. Here row 1, row 2 and row 3 represent the MSE, energy sum without and with weighting parameters, respectively. As earlier the LL3 values are scaled suitably in all cases to observe the trends.

6.3.2 Blind model. A similar experimental set, as in non blind model, is used for the blind model for nonorthonormal wavelet kernels as described in Eq. (41). The correlation coefficients, average pattern graphs for various wavelet kernels and ten different subbands are presented in TABLE 3, Fig. 8 and Fig. 9, respectively, without and with consideration of the weighting parameters. While most of the kernels show good correlations, there are occasional outliers including value of 5/3 kernel for LH3 subband in the blind model. This is due to the non-linear nature of the blind embedding algorithm (refer Section 3.2) when the model is partially effective. 
It is observed that bi-orthogonal wavelets strongly support the propositions, whereas an occasional deviation is noticed for $\mathrm{MH}$ and $\mathrm{MQ}$ wavelet kernels due its non-linear activity within the transform. However, the general behavioural pattern is maintained in all four non-orthonormal wavelets, ensures the propositions' realization in embedding distortion performance of the generalized watermarking schemes.

\subsection{Discussions}

Our experiments shows that the proposed hypothesis of a universal embedding distortion model for wavelet based watermarking algorithms has strong correlations between sum of energy and MSE for orthonormal and non-orthonormal wavelet kernels. During the experiments we also noted our observations on the influence of the chosen logo as well as performance variation in case of non blind and blind algorithms as discussed below.

In our experiments a $64 \times 64$ binary logo were used in all experiments. In case of non blind algorithms the logo was repeatedly embedded on all coefficients in a selected subband for consistent and uniform measurement of the embedding noise. This is also true for the blind watermarking scheme where number of coefficients to be embedded are fixed (one in every $3 \times 3$ window) to size of the host image not the logo. For this reason we note that the size of the watermark or logo does not have an impact on the result obtained. Additionally, our previous experiments suggest that type of logo has negligible impact on the watermarking distortion [13] and therefore choice of a logo has infinitesimal or no impact in this work.

We also observed differences in distortion performance model for non blind model which has stronger correlation compared to the blind model, i.e., non-blind watermarking schemes follow the model more accurately compared to the blind algorithms. This is largely due to the fact non-blind embedding methods are linear while the blind algorithms rely on a non-linear median operation in choosing the embedding coefficients (refer Section 3.2). Our propositions considered this fact and appropriately described the proportionality in Eq. (27) for non-blind algorithms but proposed upper bounds for blind scheme as referred in Eq. (28) and Eq. (29). Therefore the blind models only provide an upper limit to MSE.

The authors believe that the proposed model will be useful in designing new wavelet based watermarking algorithms with improved imperceptibility and robustness. This work will help the algorithmic designer to understand the impact of various input parameters, including, wavelet kernels, coefficient selection, subband choices or embedding methodology on embedding distortion. Tuning of these parameter can help in improving algorithmic robustness. For example, bi-orthogonal 5/3 and non-linear quincunx kernel has lower weighting parameters (refer TABLE 1) which is an indication that for the same watermarking strength the distortion will be lower if one chooses these kernels compared to other wavelets, e.g., orthonormal. In other words for similar MSE/PSNR, choice of these wavelet kernel will improve the robustness. Similarly, one can optimise other parameters based on the final application. This manuscript concentrates only on proposing the embedding distortion models and considers designing robust watermarking algorithm that uses this model as future work.

\section{CONCLUSIONS}

A universal embedding distortion performance model is presented in this paper for wavelet based watermarking schemes. First we have proposed models for orthonormal wavelet bases, which is then extended to nonorthonormal wavelet kernels such as biorthogonal and non-linear wavelets. The current model suggests that the MSE of the watermarked image is directly proportional to the weighted sum of energy of the modification values of the selected wavelet coefficients and this proposition is valid for orthonormal as well as non-orthonormal wavelet kernels. In the case of the non-orthonormal wavelet bases a weighting parameter is introduced and it is computed emperically for different non-orthonormal wavelet bases whereas in the case of orthonormal wavelets, these weighting parameters are set to unity. This universal model is verified by extensive experimental 
simulations with a wide range of wavelet kernels. Such a model is useful to optimize the input parameters, i.e., wavelet kernel or subband selection or the host coefficient selection in wavelet based watermarking schemes.

\section{ACKNOWLEDGMENTS}

We acknowledge the support of the UK Engineering and Physical Research Council, through a Dorothy Hodgkin Postgraduate Award.

\section{REFERENCES}

[1] C. Abhayaratne. 2011. Reversible wavelet filter banks with side informationless spatially adaptive low-pass filters. fournal of Electronic Imaging 20, 3 (2011), 033012-16.

[2] C. Abhayaratne and D. Bhowmik. 2011. Scalable Watermark Extraction for Real-time Authentication of JPEG2000 Images. Fournal of Real-Time Image Processing 6, 4 (2011), 19 pages.

[3] G. C. K. Abhayaratne and H. Heijmans. 2003. A novel morphological subband decomposition scheme for 2D+t wavelet video coding. In Proc. Int'l Symp. on Image and Signal Processing and Analysis, Vol. 1. 239-244.

[4] M.D. Adams and F. Kossentni. 2000. Reversible integer-to-integer wavelet transforms for image compression: performance evaluation and analysis. IEEE Trans. Image Processing 9, 6 (Jun 2000), 1010-1024. https://doi.org/10.1109/83.846244

[5] F. Alenizi, F. Kurdahi, A. M Eltawil, and A. K. Al-Asmari. 2019. Hybrid pyramid-DWT-SVD dual data hiding technique for videos ownership protection. Multimedia Tools and Applications 78, 11 (2019), 14511-14547.

[6] M. Amini, M. O. Ahmad, and M.N.S. Swamy. 2017. A new locally optimum watermark detection using vector-based hidden Markov model in wavelet domain. Signal Processing 137 (2017), 213-222.

[7] M. Amini, M. O. Ahmad, and M. N. S. Swamy. 2018. A Robust Multibit Multiplicative Watermark Decoder Using a Vector-Based Hidden Markov Model in Wavelet Domain. IEEE Transactions on Circuits and Systems for Video Technology 28, 2 (Feb 2018), 402-413.

[8] M. Amini, H. Sadreazami, M. O. Ahmad, and M.N.S. Swamy. 2017. A hidden Markov model-based blind detector for multiplicative watermarking. In IEEE Int'l Midwest Symposium on Circuits and Systems (MWSCAS). 611-614.

[9] M. Barni, F. Bartolini, and A. Piva. 2001. Improved wavelet-based watermarking through pixel-wise masking. IEEE Trans. Image Processing 10, 5 (May 2001), 783-791.

[10] G. Bhatnagar and Q.M. J. Wuand B. Raman. 2012. Robust gray-scale logo watermarking in wavelet domain. Computers \& Electrical Engineering (2012). https://doi.org/10.1016/j.compeleceng.2012.02.002

[11] D. Bhowmik and C. Abhayaratne. 2008. A Generalised Model for Distortion Performance Analysis of Wavelet based Watermarking. In Proc. Int'l Workshop on Digital Watermarking (IWDW'08), Lect. Notes in Comp. Sci. (LNCS), Vol. 5450. 363-378.

[12] D. Bhowmik and C. Abhayaratne. 2009. Embedding distortion modeling for non-orthonormal wavelet based watermarking schemes. In Proc. SPIE Wavelet App. in Industrial Processing VI, Vol. 7248. 72480K (12 Pages).

[13] D. Bhowmik and C. Abhayaratne. 2014. On Robustness Against JPEG2000: A Performance Evaluation of Wavelet-Based Watermarking Techniques. Multimedia Syst. 20, 2 (2014), 239-252.

[14] D. Bhowmik and C. Abhayaratne. 2016. Quality scalability aware watermarking for visual content. IEEE Transactions on Image Processing 25,11 (2016), 5158-5172.

[15] D. Bhowmik, M. Oakes, and C. Abhayaratne. 2016. Visual attention-based image watermarking. IEEE Access 4 (2016), $8002-8018$.

[16] P. Chen and J. W Woods. 2004. Bidirectional MC-EZBC with lifting implementation. Circuits and Systems for Video Technology, IEEE Transactions on 14, 10 (2004), 1183-1194.

[17] T.-S. Chen, J. Chen, and J.-G. Chen. 2003. A simple and efficient watermarking technique based on JPEG2000 codec. In Proc. Int'l Symp. on Multimedia Software Eng. 80-87.

[18] X. Cui, Y. Niu, X. Zheng, and Y. Han. 2018. An optimized digital watermarking algorithm in wavelet domain based on differential evolution for color image. Plos one 13, 5 (2018), e0196306.

[19] I. Daubechies. 1988. Orthonormal bases of compactly supported wavelets. Communications on Pure and Applied Mathematics 41, 7 (1988), 909-996.

[20] I. Daubechies. 1990. The wavelet transform, time-frequency localization and signal analysis. IEEE Transactions on Information Theory 36 , 5 (Sep 1990), 961-1005. https://doi.org/10.1109/18.57199

[21] I. Daubechies and W. Sweldens. 1998. Factoring Wavelet Transforms into Lifting Steps. Fournal of Fourier Anal. Appl. 4, 3 (1998), $245-267$.

[22] F. Dufaux, S. J. Wee, J. G. Apostolopoulos, and T. Ebrahimi. 2004. JPSEC for secure imaging in JPEG2000. In Proc. SPIE Appl. of Digital Image Processing XXVII, Vol. 5558-1. 319-330.

[23] M. Ejima and A. Miyazaki. 2001. On the evaluation of performance of digital watermarking in the frequency domain. In Proc. IEEE ICIP, Vol. 2. 546-549. 
0:18 - Bhowmik and Abhyaratne, et al.

[24] M. Fujiyoshi and H. Kiya. 2004. An image-quality guaranteed method for quantization-based watermarking using a DWT. In Proc. IEEE ICIP, Vol. 4. 2629-2632.

[25] Q. Gong and H. Shen. 2005. Toward Blind Logo Watermarking in JPEG-Compressed Images. In Proc. Int'l Conf. on Parallel and Distributed Comp., Appl. and Tech., (PDCAT). 1058-1062.

[26] F. J. Hampson and J.-C Pesquet. 1996. A Nonlinear Subband Decomposition with Perfect Reconstruction. In Proc. IEEE ICASSP, Vol. 3. $1523-1526$.

[27] H. Heijmans and J. Goutsias. 2000. Nonlinear multiresolution signal decomposition schemes: Part II: Morphological wavelets. IEEE Trans. Image Processing 9, 11 (Nov. 2000), 1897-1913.

[28] F. Huo and X. Gao. 2006. A Wavelet Based Image Watermarking Scheme. In Proc. IEEE ICIP. 2573-2576.

[29] C. Jin and J. Peng. 2006. A Robust Wavelet-based Blind Digital Watermarking Algorithm. Information Technology fournal 5, 2 (2006), $358-363$.

[30] J. R. Kim and Y. S. Moon. 1999. A Robust Wavelet-Based Digital Watermarking Using Level-Adaptive Thresholding. In Proc. IEEE ICIP, Vol. 2. 226-230.

[31] H. G. Koumaras. 2008. Subjective video quality assessment methods for multimedia applications. Technical Report ITU-R BT.500-11. Geneva, Switzerland.

[32] P. Kumsawat, K. Attakitmongcol, and A. Srikaew. 2005. A new approach for optimization in image watermarking by using genetic algorithms. IEEE Transactions on Signal Processing 53, 12 (2005), 4707-4719.

[33] D. Kundur and D. Hatzinakos. 1998. Digital Watermarking using Multiresolution wavelet decomposition. In Proc. IEEE ICASSP, Vol. 5. 2969-2972.

[34] O. Kwon and C. Lee. 2001. Objective method for assessment of video quality using wavelets. In Proc. IEEE Int'l Symp. on Industrial Electronics (ISIE 2001)., Vol. 1. 292-295.

[35] X.-L. Liu, C.-C. Lin, and S.-M. Yuan. 2016. Blind dual watermarking for color images' authentication and copyright protection. IEEE Trans. Circ. and Syst. for Video Tech 28, 5 (2016), 1047-1055.

[36] N. M Makbol, B. E. Khoo, and T. H Rassem. 2016. Block-based discrete wavelet transform-singular value decomposition image watermarking scheme using human visual system characteristics. IET Image Processing 10, 1 (2016), 34-52.

[37] S. Mallat. 1999. A wavelet tour of signal processing. Academic press.

[38] P. Meerwald. 2001. Quantization Watermarking in the JPEG2000 Coding Pipeline. In Proc. Int'l Working Conf. on Comms. and Multimedia Security. 69-79.

[39] P. Meerwald and A. Uhl. 2008. Scalability Evaluation of Blind Spread-Spectrum Image Watermarking. In Proc. Int'l Workshop on Digital Watermarking (IWDW'08), Lect. Notes in Comp. Sci. (LNCS), Vol. 5450. 61-75.

[40] A. Piper, R. Safavi-Naini, and A. Mertins. 2005. Resolution and quality scalable spread spectrum image watermarking. In Proc. 7th workshop on Multimedia and Security: MM\&Sec'05. 79-90.

[41] V. Saxena, M. Gupta, and D. T. Gupta. 2013. A Wavelet-Based Watermarking Scheme for Color Images. The IUP fournal of Telecommunications 5, 2 (Oct. 2013), 56-66.

[42] Y. Tan, J. Qin, X. Xiang, W. Ma, W. Pan, and N. N. Xiong. 2019. A Robust Watermarking Scheme in YCbCr Color Space Based on Channel Coding. IEEE Access 7 (2019), 25026-25036.

[43] D. Taubman and M. Marcellin. 2012. FPEG2000 Image Compression Fundamentals, Standards and Practice: Image Compression Fundamentals, Standards and Practice. Vol. 642. Springer Science \& Business Media.

[44] V. S. Verma and J. R. Kumar. 2014. Improved watermarking technique based on significant difference of lifting wavelet coefficients. Signal, Image and Video Processing (2014), 1-8.

[45] M. Vetterli and J. Kovačevic. 1995. Wavelets and subband coding. Prentice-Hall, Inc., Upper Saddle River, NJ, USA.

[46] Z. Wang, A. C. Bovik, H. R. Sheikh, and E. P. Simoncelli. 2004. Image quality assessment: from error visibility to structural similarity. IEEE Trans. Image Processing 13, 4 (April 2004), 600-612.

[47] A. B. Watson. 1993. Visual optimization of DCT quantization matrices for individual images. In Proc. American Institute of Aeronautics and Astronautics (AIAA) Computing in Aerospace, Vol. 9. 286-291.

[48] X. Xia, C. G. Boncelet, and G. R. Arce. 1998. Wavelet transform based watermark for digital images. Optic Express 3, 12 (Dec. 1998), 497-511.

[49] L. Xie and G. R. Arce. 1998. Joint Wavelet Compression and Authentication Watermarking. In Proc. IEEE ICIP, Vol. 2. 427-431.

[50] Z. Zhang and Y. L. Mo. 2001. Embedding strategy of image watermarking in wavelet transform domain. In Proc. SPIE Image Compression and Encryption Tech., Vol. 4551-1. 127-131. 


\section{A APPENDIX: PROOF OF PROPOSITION 1}

PRoof. Discrete wavelet transforms can be realized with a filter bank or lifting scheme based factoring. In both the cases the wavelet decomposition and the reconstruction can be represented by a polyphase matrix [21]. The inverse DWT can be defined by a synthesis filter bank using the polyphase matrix

$$
M^{\prime}(z)=\left(\begin{array}{ll}
h_{e}^{\prime}(z) & h_{o}^{\prime}(z) \\
g_{e}^{\prime}(z) & g_{o}^{\prime}(z)
\end{array}\right),
$$

where $h^{\prime}(z)$ represents the low pass filter coefficients and $g^{\prime}(z)$ is the high pass filter coefficients and the subscripts $e$ and $o$ denote even and odd indexed terms, respectively. Now the transform domain coefficient $y$ can be re-mapped into input signal $x$ as bellow:

$$
\left(\begin{array}{l}
x_{e}(z) \\
x_{o}(z)
\end{array}\right)=\left(\begin{array}{ll}
h_{e}^{\prime}(z) & h_{o}^{\prime}(z) \\
g_{e}^{\prime}(z) & g_{o}^{\prime}(z)
\end{array}\right)\left(\begin{array}{l}
y_{e}(z) \\
y_{o}(z)
\end{array}\right)
$$

Assuming $\Delta y$ is the noise introduced in wavelet domain and $\Delta x$ is the modified signal after the inverse transform, we can define the relationship between the noise in the wavelet coefficient and the noise in the modified signal using the following equations. From Eq. (44) we can write

$$
\left(\begin{array}{l}
x_{e}(z)+\Delta x_{e}(z) \\
x_{o}(z)+\Delta x_{o}(z)
\end{array}\right)=\left(\begin{array}{cc}
h_{e}^{\prime}(z) & h_{o}^{\prime}(z) \\
g_{e}^{\prime}(z) & g_{o}^{\prime}(z)
\end{array}\right)\left(\begin{array}{l}
y_{e}(z)+\Delta y_{e}(z) \\
y_{o}(z)+\Delta y_{o}(z)
\end{array}\right) .
$$

From Eq. (44) and Eq. (45) using the Linearity property of the $Z$-transform of the filter coefficients and signals in the polyphase matrix we can get,

$$
\begin{aligned}
x_{e}(z)+\Delta x_{e}(z) & =h_{e}^{\prime}(z)\left(y_{e}(z)+\Delta y_{e}(z)\right)+h_{o}^{\prime}(z)\left(y_{o}(z)+\Delta y_{o}(z)\right), \\
h_{e}^{\prime}(z) y_{e}(z)+h_{o}^{\prime}(z) y_{o}(z)+\Delta x_{e}(z) & =h_{e}^{\prime}(z) y_{e}(z)+h_{e}^{\prime}(z) \Delta y_{e}(z)+h_{o}^{\prime}(z) y_{o}(z)+h_{o}^{\prime}(z) \Delta y_{o}(z), \\
\Delta x_{e}(z) & =h_{e}^{\prime}(z) \Delta y_{e}(z)+h_{o}^{\prime}(z) \Delta y_{o}(z) .
\end{aligned}
$$

Similarly $\Delta x_{o}(z)$ can be obtained and written as

$$
\Delta x_{o}(z)=g_{e}^{\prime}(z) \Delta y_{e}(z)+g_{o}^{\prime}(z) \Delta y_{o}(z) .
$$

Combining Eq. (46) and Eq. (47), finally we can write the polyphase matrix form of the noise in the output signal:

$$
\left(\begin{array}{l}
\Delta x_{e}(z) \\
\Delta x_{o}(z)
\end{array}\right)=\left(\begin{array}{cc}
h_{e}^{\prime}(z) & h_{o}^{\prime}(z) \\
g_{e}^{\prime}(z) & g_{o}^{\prime}(z)
\end{array}\right)\left(\begin{array}{l}
\Delta y_{e}(z) \\
\Delta y_{o}(z)
\end{array}\right) .
$$

Recalling the energy conservation as stated in Eq. (48) we can conclude that

$$
\begin{aligned}
\sum\left|\Delta x_{e}\right|^{2}+\sum\left|\Delta x_{o}\right|^{2} & =\sum\left|\Delta y_{e}\right|^{2}+\sum\left|\Delta y_{o}\right|^{2}, \\
\sum_{n}|\Delta x[n]|^{2} & =\sum_{k}|\Delta y[k]|^{2} .
\end{aligned}
$$

This proves Proposition 1. 

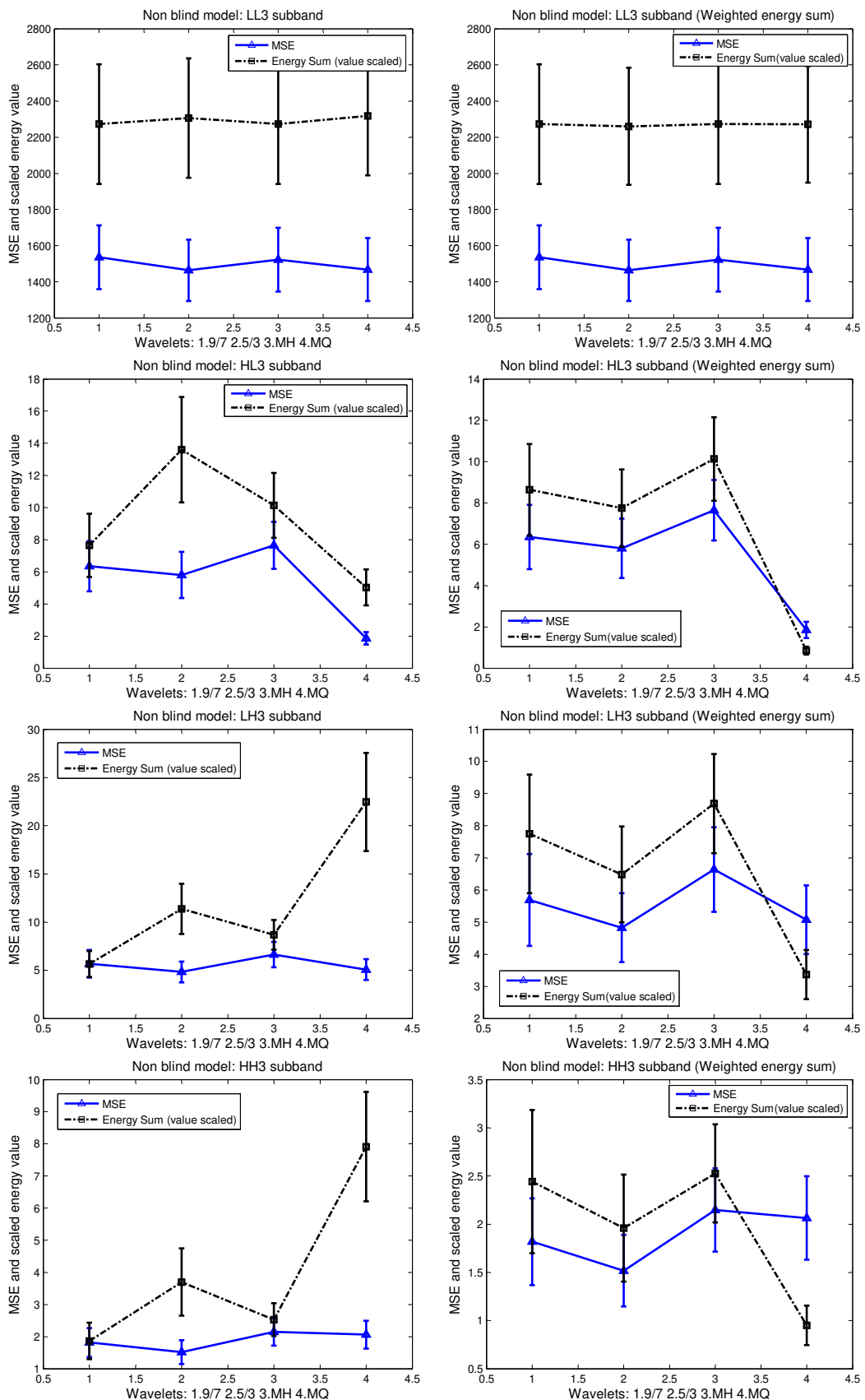

Fig. 6. Watermark embedding (non blind) performance graph for different subbands. Four different wavelet kernels used here: 1. 9/7, 2. 5/3, 3. MH and 4. MQ. Subbands are shown top to bottom for LL3, HL3, LH3, HH3, respectively. Column 1 and column 2 represent the results without and with considering the weighting parameter, while calculating the energy sum, respectively. 

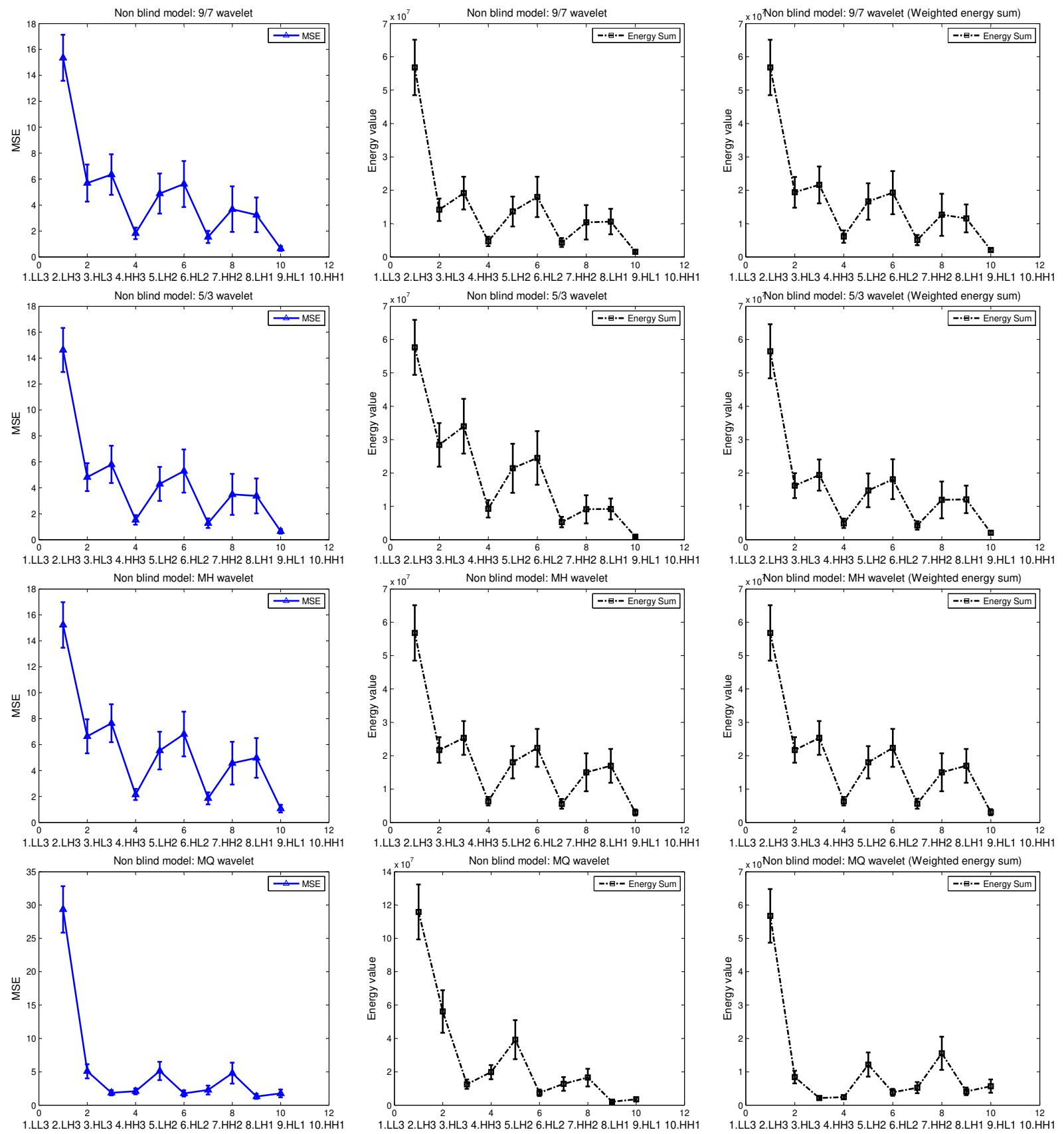

Fig. 7. Watermark embedding (non blind) performance graph for various wavelets in different subband. Wavelet kernels are shown top to bottom as 1.9/7, 2. 5/3,3. MH and 4. MQ, respectively. Column 1, column 2 and column 3 represent the MSE, energy sum without and with weighting parameters, respectively. 

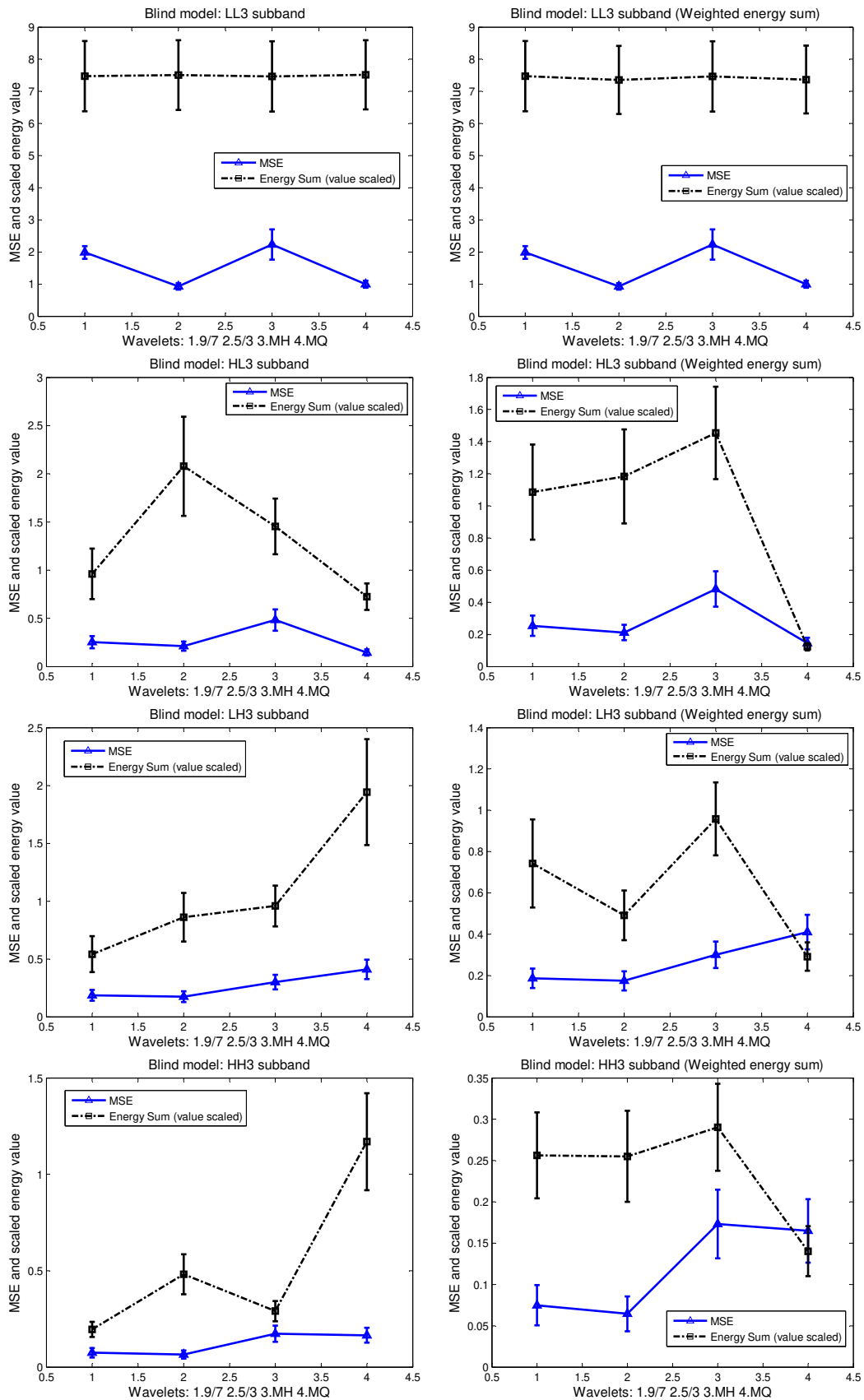

Fig. 8. Watermark embedding (blind) performance graph for different subbands. Four different wavelet kernels used here: 1. 9/7, 2. 5/3, 3. MH and 4. MQ. Subbands are shown top to bottom as LL3, HL3, LH3 and HH3, respectively. Column 1 and column 2 represent the results without and with considering the weighting parameter, while calculating the energy sum, respectively. 

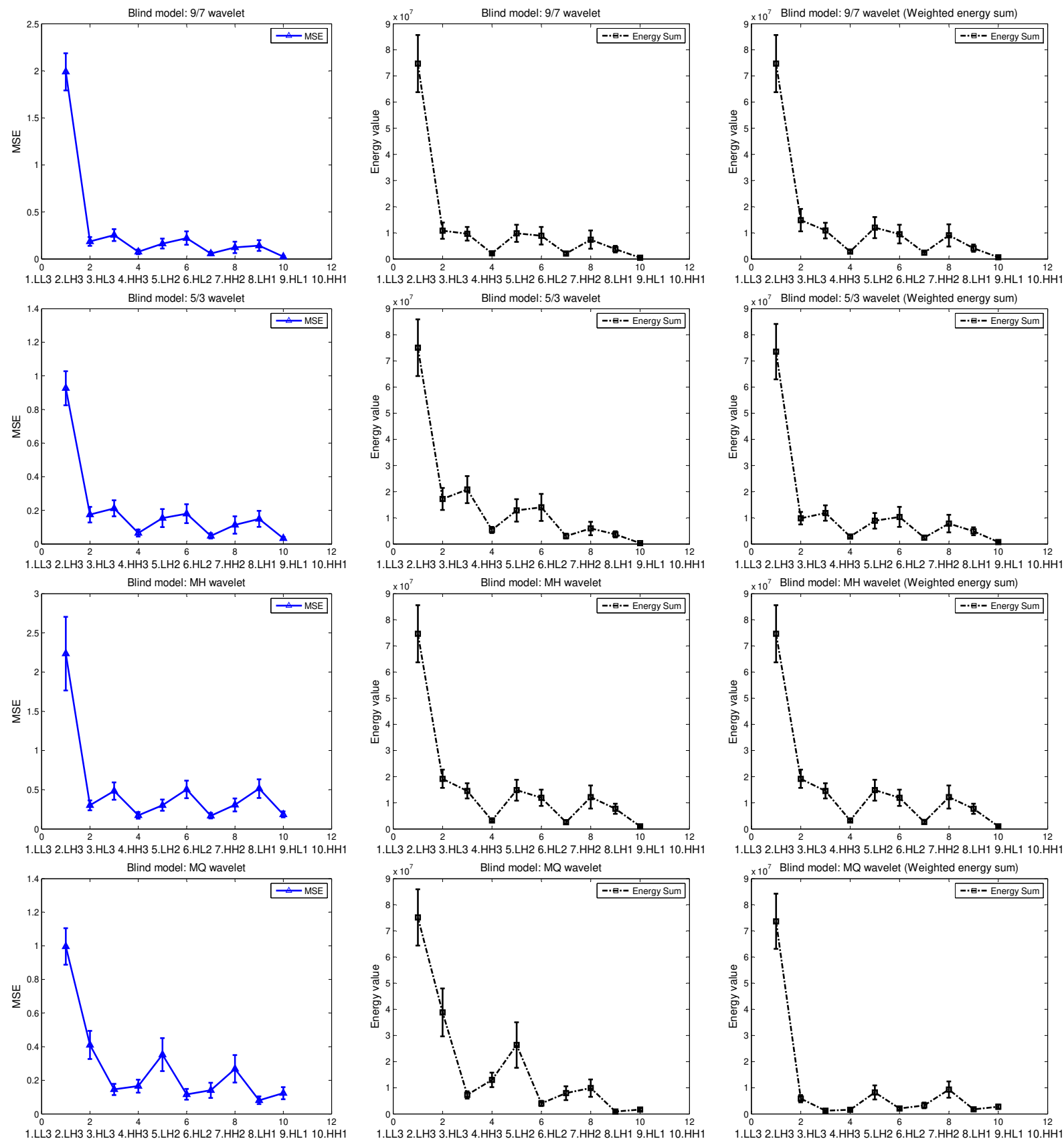

Fig. 9. Watermark embedding (blind) performance graph for various wavelets in different subband. Wavelet kernels are shown top to bottom as 1.9/7, 2. 5/3,3. MH and 4. MQ, respectively. Coulmn 1, column 2 and column 3 represent the MSE, energy sum without and with weighting parameters, respectively. 